\title{
Geometrical Optics Convergence Coefficient for Whistler Propagation
}

\author{
G. McK. Allcock \\ Contribution From the Department of Scientific and Industrial Research, Physics and Engineering Laboratory, \\ Lower Hutt, New Zealand
}

(Received May 25, 1964)

\begin{abstract}
It is shown that the formula, given by Crary [1964] for the convergence coefficient for whistler signals propagated by multiple reflection between a convex earth and a concave ionosphere, can be considerably simplified. The simplified formula shows that the convergence coefficient for a given number of reflections is independent of the height of the ionosphere, and that antipodal focusing would be expected. The convergence takes place entirely in a horizontal direction transverse to the plane of propagation.

A very simple first-order approximation to the formula is found to be adequate for most practical calculations. A second-order approximation shows that the convergence coeficient for a given propagation distance is almost independent of the number of reflections.
\end{abstract}

\section{Introduction}

Crary [1964] has recently derived an expression for the convergence coefficient in the ray theory equations for the field strength near the surface of a sharply bounded ionosphere due to a radio transmitter on the ground. His paper has served a useful purpose in showing that the convergence coefficient is indeed close to unity. This, as Crary points out, would intuitively be expected, since there are an equal number of reflections from the concave ionosphere and the convex ground. However, equation (17) of Crary's paper, which he has used for his illustrative calculations, is by no means in its simplest form, which we here derive.

In figure 1 we have redrawn the first half-hop portion of Crary's figure 2, labeling the ground-based transmitter position $G$, the point of incidence of the ray on the ionosphere $I$, and the center of the earth $O$. We now construct $O Z$ perpendicular to $I G$, $Z Y$ perpendicular to $O G$, and $I X$ perpendicular to $O G$. Then

$$
O X=(a+h) \cos \left(\frac{\gamma}{2 n+1}\right)
$$

Also, since $/ O Z Y=/ Z G^{V}=\phi$,

$$
O Y=O Z \sin \phi=a \sin ^{2} \phi .
$$

Combining (1) and (2),

$$
\begin{aligned}
(a+h) \cos \left(\frac{\gamma}{2 n+1}\right)-a \sin ^{2} \phi & =O X-O Y=X Y \\
& =I Z \cos \phi \\
& =(a+h) \cos \theta_{n} \cos \phi .
\end{aligned}
$$

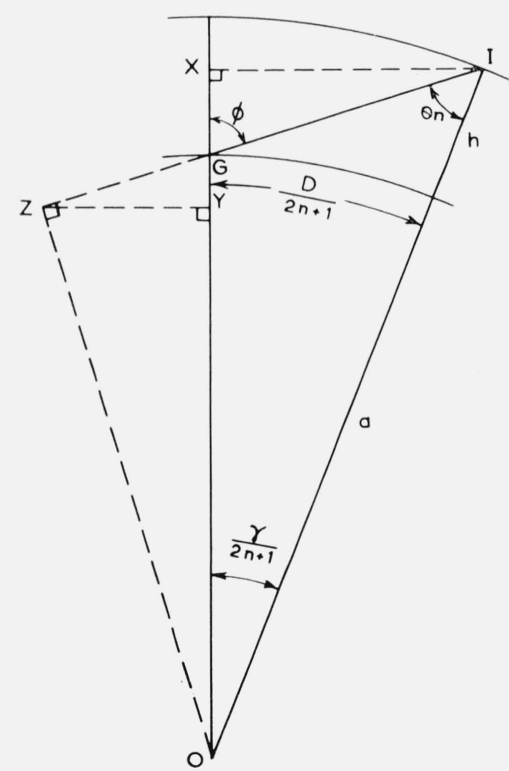

FiguRE 1. Geometry for simplification of convergence coefficient formula.

Also,

$$
a \sin \phi=O Z=(a+h) \sin \theta_{n} .
$$

We can now substitute (3) and (4) in Crary's equation (17), which reads 


$$
\begin{array}{r}
\alpha_{\omega h}=\frac{a}{(a+h) \sin \theta_{n}}\left\{\frac{(2 n+1) \sin \phi \tan \phi \sin \left(\frac{\gamma}{2 n+1}\right)\left[(a+h) \cos \left(\frac{\gamma}{2 n+1}\right)-a \sin ^{2} \phi\right]}{(a+h) \sin \gamma \cos \theta_{n}}\right\}^{1 / 2} \\
=\frac{1}{\sin \phi}\left\{\frac{(2 n+1) \sin ^{2} \phi \sin \left(\frac{\gamma}{2 n+1}\right)(a+h) \cos \theta_{n} \cos \phi}{\cos \phi(a+h) \sin \gamma \cos \theta_{n}}\right\}^{1 / 2}
\end{array}
$$

which reduces to

$$
\alpha_{\omega h}=\left\{\frac{(2 n+1) \sin \left(\frac{\gamma}{2 n+1}\right)}{\sin \gamma}\right\}^{1 / 2} .
$$

This formula can also be derived analytically, though more laboriously, from Crary's equation (17), by applying the sine and cosine rules to the triangle $I G O$, and thence expressing the functions of $\phi$ and $\theta_{n}$ in terms of functions of $\left(\frac{\gamma}{2 n+1}\right)$.

\section{Approximate Formulas for Distances Less Than $6000 \mathrm{~km}$}

We can further simplify (5) for use at short distances. Upon reference to figure 1, it can be seen that, for the case of VLF propagation where $h$ is 90 $\mathrm{km}$ or less, $\cos \left(\frac{\gamma}{2 n+1}\right)$ will always be greater than $\left(\frac{a}{a+h}\right)$, except when propagation is tangential to the earth's surface. Therefore $\left(\frac{\gamma}{2 n+1}\right)$ will always be less than 0.168 radian $\left(9.6^{\circ}\right)$, and thus the approximation

$$
\sin \left(\frac{\gamma}{2 n+1}\right) \approx \frac{\gamma}{(2 n+1)}
$$

will always be correct to within 0.5 percent. We can also write

$$
\sin \gamma \approx \gamma-\frac{\gamma^{3}}{6}
$$

to 1 percent accuracy for $\gamma$ less than 1 radian, i.e., for great-circle distances $D$ less than $6370 \mathrm{~km}$. Using approximations (6) and (7), (5) becomes

$$
\begin{aligned}
& \alpha_{\omega h} \approx\left\{\frac{1}{1-\frac{\gamma^{2}}{6}}\right\}^{1 / 2} \\
& \approx 1+\frac{\gamma^{2}}{12} \\
& \approx 1+\frac{1}{12}\left(\frac{D}{a}\right)^{2} .
\end{aligned}
$$

The nature of the approximations is such that (8) will be correct to within about 1 percent for distances less than $6000 \mathrm{~km}$.
It is seen from (8) that, to a first approximation, the convergence coefficient is independent of $n$, the number of reflections. A close estimate of the variation of $\alpha_{w h}$ with $n$ is obtained by taking a second approximation

$$
\sin \left(\frac{\gamma}{2 n+1}\right) \approx\left(\frac{\gamma}{2 n+1}\right)-\frac{1}{6}\left(\frac{\gamma}{2 n+1}\right)^{3}
$$

instead of (6), and proceeding as before. This time we get

$$
\begin{aligned}
& \alpha_{\omega h} \approx 1+\frac{n(n+1) \gamma^{2}}{3(2 n+1)^{2}} \\
& \approx 1+\frac{n(n+1)}{3(2 n+1)^{2}}\left(\frac{D}{a}\right)^{2}
\end{aligned}
$$

which is equivalent to (8) for $n=\infty$. The error in using this approximation is less than 0.1 percent for distances up to $4000 \mathrm{~km}$, and less than 0.5 percent at $6000 \mathrm{~km}$. The dependence of $\alpha_{\omega h}$ on $n$ is expressed by the term $\frac{n(n+1)}{3(2 n+1)^{2}}$, which is almost invariant, having the value 0.07407 for $n=1$, and increasing slowly to 0.08333 for $n=\infty$. The sole exception is the case where $n=0$, i.e., the transmitter and receiver are in direct line-of-sight. Here, as would be expected from elementary considerations, $\frac{n(n+1)}{3(2 n+1)^{2}}=0$, and $\alpha_{\omega h}=1$ exactly.

The results of calculations of $\alpha_{\omega h}$ for distances up to $6000 \mathrm{~km}$ are shown in figure 2. This shows graphically the virtual independence of $\alpha_{\omega h}$ on $n$.

\section{Discussion}

One feature which is shown by (5), but which was not evident in Crary's equation (17), is that the convergence coefficient in the whistler case is independent of the height of the ionosphere, except insofar as the minimum value of $n$ is determined by the tangency condition $\phi=90$. For a daytime ionospheric height of $70 \mathrm{~km}$, the maximum values of $D$ for $n=0,1$, and 2 are marked by vertical stops in figure 2 .

Equation (5) can also be derived $a b$ initio by considering the geometry of a spreading bundle of rays with rectangular cross section, as used by Rawer [1952]. This approach shows that the net convergence in the whistler case takes place entirely in a horizontal direction transverse to the plane of propagation. By contrast, for the case of ground- 


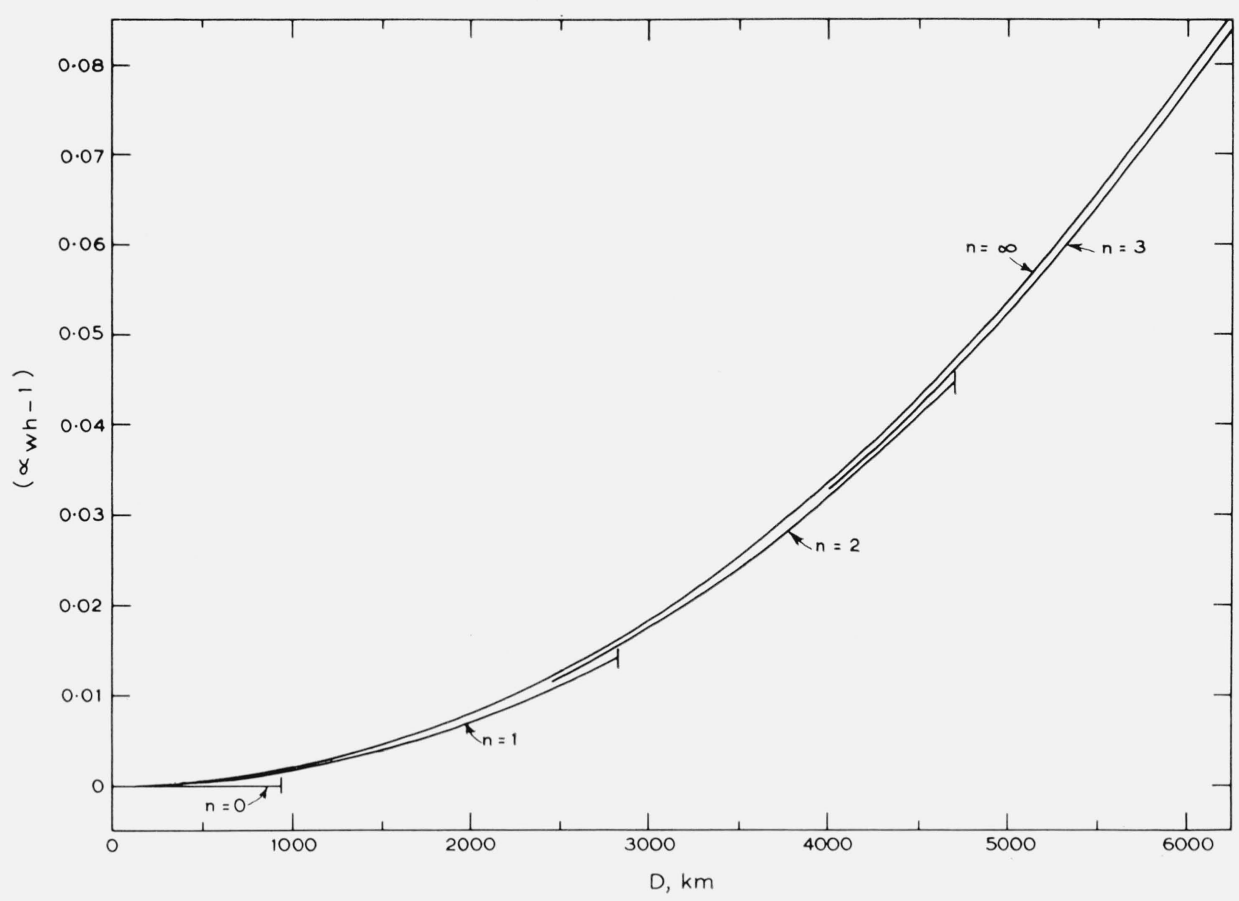

Figure 2. Convergence coefficient as a function of propagation distance.

to-ground transmission, it is seen that a term similar to (5) appears in the formula for the convergence coefficient. [Crary's equation (1).] The remainder of the terms in the ground-to-ground formula, viz,

$$
\left(1+\frac{h}{a}\right)\left[\frac{\left(1+\frac{h}{a}\right)-\cos (D / 2 a n)}{(1+h / a) \cos (D / 2 a n)-1}\right]^{1 / 2},
$$

state the amount of convergence taking place within the plane of propagation, in a direction perpendicular to the ray path.

An examination of (5) shows that $\alpha_{\omega h}$ becomes infinite when $\sin \gamma=0$, provided that $\sin$ $\left(\frac{\gamma}{2 n+1}\right) \neq 0$, i.e., infinities occur for $\gamma=k \pi \quad(k=1$, $2,3 \ldots$. . . The principal value $\gamma=\pi$ represents the case of antipodal focusing, already well known for ground-to-ground propagation.
Finally, it should be emphasized that the above discussion has been confined to geometrical optics. No account has been taken of diffraction near grazing incidence, nor of other factors which enter into the calculation of field strength, such as the reflection coefficients of the ionosphere and the earth's surface, and the polar diagram of the transmitting antenna. This aspect of the problem has been discussed thoroughly by Wait [1961].

\section{References}

Crary, J. H. (1964), Geometrical optics convergence coefficient for the whistler case, Radio Sci. J. Res. NBS/USNCURSI 68D, No. 2, 211-214.

Rawer, K. (1952), Calculation of sky-wave field strength, Wireless Engr. 29, 287-301.

Wait, J. R. (1961), A diffraction theory for LF sky wave propagation, J. Geophys. Res. 66, 1713-1724.

(Paper 68D11-415) 